\title{
Symmetries of Quasi-Values
}

\author{
Ales A. Kubena ${ }^{1}$ and Peter Franek ${ }^{2}$ \\ 1 Institute of Information Theory and Automation of the ASCR, \\ Pod Vodarenskou vezi 4, 182 08, Prague, Czech Republic \\ kubena@utia.cas.cz, \\ 2 Institute of Information Technologies, Czech Technical University, Thakurova 9, \\ Prague 160 00, Czech Republic \\ peter.franek@fit.cvut.cz
}

\begin{abstract}
According to Shapley's game-theoretical result, there exists a unique game value of finite cooperative games that satisfies axioms on additivity, efficiency, null-player property and symmetry. The original setting requires symmetry with respect to arbitrary permutations of players. We analyze the consequences of weakening the symmetry axioms and study quasi-values that are symmetric with respect to permutations from a group $G \leq S_{n}$. We classify all the permutation groups $G$ that are large enough to assure a unique $G$-symmetric quasi-value, as well as the structure and dimension of the space of all such quasi-values for a general permutation group $G$.

We show how to construct $G$-symmetric quasi-values algorithmically by averaging certain basic quasi-values (marginal operators).
\end{abstract}

\section{Introduction}

A cooperative game is an assignment of a real number to each subset of a given set of players $\Omega$. This illustrates an economic situation where a coalition profit depends on the involved players in a generally non-aditive way. Several approaches deal with the question of redistributing the generated profit to the individual players in a stable or in a "fair" way. The mathematical theory of cooperative games was developed in forties by Neumann and Morgenstern [17. Values of games provide a tool for evaluating the contributions of the individual players such that certain natural axioms are satisfied. The most famous value is the Shapley value introduced in 1953 [22] that exists and is unique for all finite sets of players.

There exist many axiomatic systems on game values such that the Shapley value is their only solution: the original Shapley's axiomatics [22], Neyman's [18], Young's 24, van den Brink's 3 and Kar's axiomatics [15. One of its important characteristics is the symmetry with respect to any permutation of players. This means, roughly speaking, that the value of a player is calculated only from his contributions to various coalitions and not from his identity. One may consider this to represent the equity of players. However, this is probably not a realistic assumption in many real-world situations where personal friendships and linkage play a major role. Some examples of values with restricted symmetry were 
studied, such as the Owen value [20] or the weighted Shapley value in [14], and the formal concept of quasi-value, where one completely relaxes any symmetry requirement, was introduced by Gilboa and Monderer in 1991 [10. It is known that for a particular player set, there exist infinitely many quasi-values.

In this work, we analyze one particular way of weakening the symmetry axiom. We suppose that a group $G$ of permutations of $\Omega$ is given and define a $G$ symmetric quasi-value to be any quasi-value symmetric wrt. all permutations in $G$. Informally, the equity of players is restricted to a group of permutations of players, not necessarily to all permutations. The group expresses the measure of symmetry. If $G$ is the full symmetry group, then the only $G$-symmetric quasivalue is the Shapley value; if $G$ is the trivial group, then it carries no symmetry requirement and each quasi-value is $G$-symmetric. Our contribution is the classification of all permutation groups $G$ of finite sets of players for which there exists a unique $G$-symmetric quasi-value. It turns out that while in the infinite setting for non-atomic games one may reduce the group of symmetries in a number of ways 16[19, in the finite setting, only few subgroups of the full permutation group assure uniqueness. Even if the group $G$ acts transitively on $\Omega$ (i.e. for any two players $a, b$, there exists a permutation $\pi \in G$ such that $\pi(a)=b)$, there may still exist many $G$-symmetric quasi-values different from the Shapley value. We also calculate the dimension of the space of all $G$-symmetric quasi-values for a general permutation group $G$.

In the second section, we give the formal definition of $G$-symmetric quasivalue and some necessary definitions from group theory, including our original definition of a supertransitive group action. In the third section, we show that the space of all $G$-symmetric quasi-values is an affine subspace of the vector space of all values, and derive a formula for its dimension. We further classify all permutation groups $G$ such that there exists a unique $G$-symmetric quasivalue. In the fourth section, we give some examples of $G$-symmetric quasi-values and show how more examples can be constructed by averaging the marginal operators. The last section (Appendix) contains the proof of an auxiliary statement from group theory that we use in the proof of Theorem 2. We postpone this technical issue to the end in order to keep the rest of the text fluent.

\section{Definitions and notation}

\subsection{Cooperative games}

Let $\Omega$ be a set of players. In this paper, we always suppose that $\Omega$ is finite.

Definition 1. A cooperative game is a function $v: 2^{\Omega} \rightarrow \mathbb{R}$ such that $v(\emptyset)=0$. A cooperative game is additive, if for all $T, R \in 2^{\Omega}, R \cap T=\emptyset$ implies $v(R \cup T)=$ $v(R)+v(T)$. We denote by $\Gamma$ the set of all cooperative games and $\Gamma_{1}$ the set of all additive cooperative games. A game value is an operator $\varphi: \Gamma \rightarrow \Gamma_{1}$. For a game value $\varphi$ and $i \in \Omega$, we define $\varphi_{i}(v):=\varphi(v)(\{i\})$.

For each game $v, \varphi(v)$ is uniquelly determined by the numbers $\varphi_{i}(v)$. Shapley theorem 22 proves the existence and uniqueness of a game value $\varphi$ assuming it satisfies the following four axioms: 
1. Linearity: $\varphi(\alpha v+\beta w)=\alpha \varphi(v)+\beta \varphi(w)$ for all $v, w \in \Gamma$ and $\alpha, \beta \in \mathbb{R}$.

2. Null-player property: if $i \in \Omega$ is a "null-player" in a game $v$, i.e. $v(R \cup\{i\})=$ $v(R)$ for each $R \subseteq \Omega$, then $\varphi_{i}(v)=0$.

3. Efficiency: $\sum_{i} \varphi_{i}(v)=v(\Omega)$ for all games $v$.

4. Symmetry (sometimes called anonymity): $\varphi(\pi \cdot v)=\pi \cdot \varphi(v)$ for every permutation $\pi$ of $\Omega$, where the game $\pi \cdot v$ is defined by $(\pi \cdot v)(R):=v\left(\pi^{-1}(R)\right)$ for any $R \subseteq \Omega$.

The value defined by these axioms is called Shapley value. Axioms 14 are independent. Gilles [11] and Schmeidler [5] give examples of values satisfying any 3 of them and not the 4th.

Any game value satisfying axioms 1 2 and 3 is called a quasi-value. In the original economic interpretation, the fourth axiom (Symmetry) is an expression of equity of all the participating players. It can be formulated in a more elegant way by the commutativity of the following diagram.

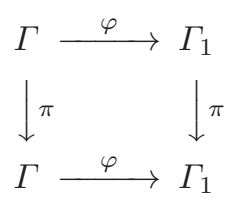

Axiom 4 requires that it commutes for each permutation of players $\pi$.

The following definition introduces the main object of our study.

Definition 2. Let $G$ by a group of permutations of $\Omega$. A G-symmetric quasi-

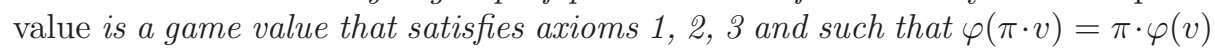
for every permutation $\pi \in G$. In other words, diagram (11) commutes for all $\pi \in G$.

Throughout this work, we will need the following basis of the space of cooperative games, introduced in Shapley's original paper [22.

Definition 3. The unanimity basis is the basis $\left\{u_{R}\right\}_{\emptyset \neq R \subseteq \Omega}$ of the vector space of all cooperative games over the set $\Omega$, defined by $u_{R}(S)=1$ if $R \subseteq S$ and 0 otherwise.

\subsection{Group theory}

We say that a group $G$ acts on the set $X$, if $G$ is a subgroup of the group $S_{X}$ of permutations of $X$. Any set $G \cdot x$ is called an orbit, or a $G$-orbit of $x$. The set of all $G$-orbits is denoted by $X / G$. The action of $G$ on $X$ is transitive, if for each $x, y \in X$, there exists a $g \in G$ such that $g \cdot x=y$. The stabilizer of a subset $A \subseteq X$ is the subgroup $G_{A}$ of all elements $g \in G$ such that $g \cdot A \subseteq A$. For a subgroup $H$ of $G, g \cdot H$ denotes a left and $H \cdot g$ a right coset of $H$ and any group $H^{\prime}=g^{-1} H g$ is conjugate to $H$.

We introduce here a definition that will help us to describe a property of permutation groups we will need later. 
Definition 4. Let $G$ be a group acting on a set $X$. We say that the action is $a$ supertransitive action, if the stabilizer $G_{A}$ of any subset $A \subseteq X$ acts transitively on $A$. A permutation group $G \subseteq S_{n}$ is supertransitive, if the stabilizer $G_{A}$ acts transitively on each $A \subseteq\{1, \ldots, n\}$.

For any $n, S_{n-1}$ may be embedded into $S_{n}$ as a set of permutations preserving one element. However, for $n=6$, there exists an embedding of $S_{5}$ into $S_{6}$ different from the standard one. This embedding $S_{5} \hookrightarrow S_{6}$ may be realized as the action of the projective linear group $P G L(2,5)$ on the projective line over $\mathbb{Z}_{5}$. The reader may find the details in the literature [7, p. 60-61], 44. We will call this embedding an exotic embedding. It is well known that such a nonstandard embedding is only one up to conjugation by an element of $S_{6}$. In this paper, we only need the property that the image of the exotic embedding is a supertransitive subgroup of $S_{6}$. This is proved in the appendix.

\section{Dimension of $G$-symmetric quasi-values}

If a quasi-value is symmetric with respect to a set of permutations, it is also symmetric with respect to any permutation they generate in $S_{\Omega}$, hence the set of all symmetries of a quasi-value is always a group. For a finite set $\Omega$ and a group $G \subseteq S_{\Omega}$ of permutations, we denote by $\mathcal{A}_{G}$ the set of all $G$-symmetric quasi-values.

We will represent $\mathcal{A}_{G}$ as a space of matrices. Each game value $\varphi$ can be represented as a map from $\Gamma$ to $\mathbb{R}^{\Omega}$ by the natural identification $\Gamma_{1} \simeq \mathbb{R}^{\Omega}$. Choosing the unanimity basis on $\Gamma$ (Def. 3) and the canonical basis $\left(e_{i}\right)_{i \in \Omega}$ on $\mathbb{R}^{\Omega}$, we may represent linear game values as matrices of the size $|\Omega| \times\left(2^{|\Omega|}-1\right)$. The null player property applied to the unanimity basis implies $\varphi\left(u_{R}\right)(\{i\})=0$ for each $i \notin R$, because such player $i$ doesn't contribute to any coalition in the game $u_{R}$. As a consequence, a matrix $A$ with elements $\left(a_{i R}\right)_{i \in \Omega, \emptyset \neq R \subseteq \Omega}$ corresponds to a linear game value satisfying the null-player-property iff $a_{i R}=$ 0 for all pairs $(i, R)$ such that $i \notin R$. Further, the game value satisfies the efficiency axiom iff for any nonempty $R \subseteq \Omega, \varphi\left(u_{R}\right)(\Omega)=1$, which translates to a constraint on matrix coefficients $\sum_{i \in R} a_{i R}=1$ for each $\emptyset \neq R \subseteq \Omega$. The $G$-symmetry of a game value requires $\varphi(g \cdot v)=g \cdot(\varphi(v))$ for any game $v$ and permutation $g \in G$, the action of $G$ on $\Gamma$ defined by $(g \cdot v)(R)=v\left(g^{-1} R\right)$. An element $u_{R}$ from the unanimity basis satisfies $\left(g \cdot u_{R}\right)(S)=u_{R}\left(g^{-1}(S)\right)=$ $u_{g R}(S)$, so the unanimity basis is invariant with respect to the group action and $g \cdot u_{R}=u_{g R}$. The symmetry axiom is equivalent to

$$
\left((g \cdot \varphi)\left(u_{R}\right)\right)(\{i\})=\left(\varphi\left(u_{g R}\right)\right)(\{i\}),
$$

for all $i \in \Omega$ and $\emptyset \neq R \subseteq \Omega$. The left-hand side is equal to $\varphi\left(u_{R}\right)\left(\left\{g^{-1} i\right\}\right)$. So, in the matrix representation of $\varphi$, the symmetry axiom translates to the condition $a_{\left(g^{-1} i\right) R}=a_{i(g R)}$, or simply $a_{i R}=a_{(g i)(g R)}$ for all $i \in \Omega, \emptyset \neq R \subseteq \Omega$ and $g \in G$.

Summarizing this, we have the following. 
Lemma 1. Choosing the unanimity basis of $\Gamma$ and the canonical basis of $\mathbb{R}^{\Omega} \simeq$ $\Gamma_{1}, \mathcal{A}_{G}$ may be identified with a set of matrices $A=\left(a_{i R}\right)$ with elements satisfying the following equations:

$-a_{i R}=0$ if $i \notin R$,

- The sum of elements in each column is 1 ,

- Matrix elements $a_{i R}$ are constant on the orbits of the $G$-action $g \cdot(i, R)=$ $(g i, g R)$.

All these conditions are linear equations for matrix elements $a_{i R}$ and they are all satisfied by the Shapley value. So, $\mathcal{A}_{G}$ is a nonempty affine space.

Theorem 1. Let $X=\{(i, R) ; i \in R \subseteq \Omega\}, \chi=\{R ; \emptyset \neq R \subseteq \Omega\}$ and let $G \subseteq S_{G}$ be a group of permutations acting on sets $X$ and $\chi$, extending naturally its action on $\Omega$. Then the dimension of $\mathcal{A}_{G}$ is $|X / G|-|\chi / G|$. Explicitly it can also be expressed as

$$
\operatorname{dim} \mathcal{A}_{G}=\left.\left(\frac{d Z_{G}}{d x_{1}}-Z_{G}\right)\right|_{(2,2 \ldots 2)}+1
$$

where $Z_{G}$ is the cycle index of the group $G$

$$
Z_{G}\left(x_{1} \ldots x_{n}\right)=\frac{1}{|G|} \sum_{\pi \in G} x_{1}^{j_{1}(\pi)} \cdots x_{n}^{j_{n}(\pi)},
$$

$j_{k}(\pi)$ denotes the number of cycles of length $k$ in the permutation $\pi$ [8, $\left.p .85\right]$.

Proof. We will identify elements of $\mathcal{A}_{G}$ with matrices as described in Lemma 1) Let $p: X \rightarrow \chi$ be the map $(i, R) \rightarrow R$. For any $x=(i, R) \in X$ and $g \in G$, $p(g x)=g(p(x))$. For $\emptyset \neq R \subseteq \Omega$, the stabilizer $G_{R}$ acts on $R$ and $R$ splits into $k_{R}$ orbits $\left\{R_{1}, \ldots, R_{k_{R}}\right\}$ with respect to this action. If $R^{\prime}=g R$, then the stabilizer of $R^{\prime}$ is $g G_{R} g^{-1}$ and $g$ maps each $G_{R^{-}}$orbit $R_{i} \subseteq R$ bijectively onto a $G_{R^{\prime}}$-orbit $R_{i}^{\prime} \subseteq R^{\prime}$. So, $k_{R}=k_{R^{\prime}}$ and $\left|R_{i}\right|=\left|R_{i}^{\prime}\right|$ for $i=1, \ldots, k_{R}$. For $m \in \chi / G$, we define $k_{m}:=k_{R}$ for any $R \in m$ and $l_{m i}=\left|R_{i}\right|$ for $i=1, \ldots, k_{m}$. These numbers are independent on the choice of $R$.

We will say that $m \in \chi / G$ contains an orbit $G x \in X / G$, if $p(x) \in m$. Each $m \in \chi / G$ contains $k_{m}$ orbits $\left\{o_{1}, \ldots, o_{k_{m}}\right\} \subseteq X / G$ and we may choose real numbers $c_{m i}$ such that $\sum_{i=1}^{k_{m}} c_{m i} l_{m i}=1$ with $k_{m}-1$ degrees of freedom. Choosing such numbers $c_{m i}$ for all $m \in \chi / G$ gives

$$
\sum_{m \in \chi / G}\left(k_{m}-1\right)=\sum_{m \in M} k_{m}-|\chi / G|=|X / G|-|\chi / G|
$$

degrees of freedom. Any such choice of $c_{m i}$ defines a matrix of game value

$$
a_{i R}=\left\{\begin{array}{l}
c_{m i} \text { if } i \in R_{i} \subseteq R \in m \\
0 \text { if } i \notin R
\end{array}\right.
$$


These are exactly matrices $A$ constant on the orbits of $X$ satisfying $\sum_{i} a_{i R}=1$ for all $R$ and $a_{i R}=0$ for all $i \notin R$. The number of degrees of freedom for the choice of $c_{m i}$ is equal to the dimension of $\mathcal{A}_{G}$. This proves the first part.

Burnside lemma [21, p. 58] enables to express the number of orbits of a group action in an explicit way. If a finite group $H$ acts on a finite set $Y$, then

$$
|Y / H|=\frac{1}{|H|} \sum_{h \in H}|\{y \in Y \mid h(y)=y\}| .
$$

A permutation $\pi \in G$ fixes those sets $R \subseteq \Omega$ that don't split any cycle of $\pi$. There exists $2^{\# \operatorname{cycles}(\pi)}$ such sets, $2^{\# \operatorname{cycles}(\pi)}-1$ of them nonempty. So,

$$
|\chi / G|=\left(\frac{1}{|G|} \sum_{\pi \in G} 2^{\# \operatorname{cycles}(\pi)}\right)-1 .
$$

Elements of $X$ fixed by $\pi$ are pairs $(i, R)$ such that $i \in R, \pi(i)=i$ and $\pi(R)=R$. There exists \# fixedpoints $(\pi) * 2^{\# \text { cycles }(\pi)-1}$ such pairs. We derived the following equation:

$$
\operatorname{dim} \mathcal{A}_{G}=\frac{1}{|G|}\left(\sum_{\pi \in G}\left(\# \operatorname{fixedpoints}(\pi) * 2^{\# \operatorname{cycles}(\pi)-1}\right)-\sum_{\pi \in G} 2^{\# \text { cycles }(\pi)}\right)+1
$$

The statement of the theorem follows from this by a direct computation.

The cycle index $Z_{G}$ is known in a more explicit form than (3) for many subgroups of $S_{n}$ and it has also been generalized and computed for finite classical groups [9].

Further, we will show for which groups $G$ the dimension of $\mathcal{A}_{G}$ is zero, i.e. for which $G$ the only $G$-symmetric quasi-value is the Shapley value. In Section 2.2. we defined a group $G \subseteq S_{\Omega}$ to be supertransitive, if the stabilizer $G_{R}$ acts transitively on $R$ for each subset $R \subseteq \Omega$. In other words, if for each $R$ and each $i, j \in R$, there exists a $g \in G$ such that $g(R)=R$ and $g \cdot i=j$. We will show that this condition is equivalent to the existence of a unique $G$-symmetric quasi-value.

Theorem 2. Let $\Omega$ be finite and $G \leq S_{\Omega}$. There exists a unique $G$-symmetric quasi-value if and only if $G$ acts supertransitively on $\Omega$. Equivalently, this is if and only one of the following conditions is satisfied:

$-G=S_{\Omega}$, the full symmetric group

- $|\Omega|>3$ and $G=A_{\Omega}$, the alternating group

- $|\Omega|=6$ and $G$ is the image of an exotic embedding $S_{5} \hookrightarrow S_{6}$ (see Section 2.2).

Proof. We will work with the matrix representation of $\mathcal{A}_{G}$, described in Lemma1] Let $\left(a_{i R}\right)$ be a matrix representing a value in $\mathcal{A}_{G}$.

If the action of $G$ on $\Omega$ is supertransitive, then for each $\emptyset \neq R \subseteq \Omega$, all elements $\{(i, R) ; i \in R\}$ lie on the same $G$-orbit, so all the corresponding matrix elements $a_{i R}$ are equal. The null-player property implies that $a_{i R}=0$ for $i \notin R$ 
and together with the efficiency condition we obtain that for each $i \in R, a_{i R}=$ $1 /|R|$. This implies uniqueness.

If the action of $G$ on $\Omega$ is not supertransitive, then there exists a nonempty subset $\tilde{R} \subseteq \Omega$ such that the stabilizer $G_{\tilde{R}}$ has not a transitive action on $\tilde{R}$. So, $\tilde{R}$ contains at least two $G_{\tilde{R}}$-orbits. We may define the matrix $a_{i R}$ as follows. In the matrix column corresponding to $\tilde{R}$ we choose $a_{i \tilde{R}}=0$ if $i \notin \tilde{R}$ and the

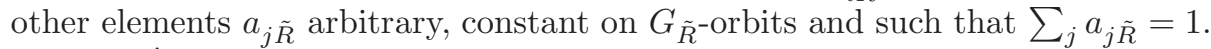
For all $R^{\prime}$ on the $G$-orbit of $R$, we define the coefficients $a_{i R^{\prime}}$ in a unique way so that they are constant on the $G$-orbits and the remaining matrix elements may be equal to elements of the original Shapley matrix. In this way, we may construct an infinite number of different $G$-symmetric quasi-values which proves that $\operatorname{dim} \mathcal{A}_{G} \geq 1$.

For the classification part, it remains to prove that the groups listed in the theorem are exactly the groups acting supertransitively on $\{1, \ldots, n\}$. The proof of this is technical and we postpone it to the Appendix (Chapter 5 ).

\section{Consequences}

\subsection{Examples}

First we give some examples of groups and $G$-symmetric quasi-values. In all these examples, we assume that the player set $\Omega$ consists of $n$ players.

Example 1. Let $G_{1}=\{\mathrm{id}\}$ be the trivial group. In this case, any quasi-value is $G_{1}$-symmetric. Consider a selector $\gamma: 2^{\Omega} \rightarrow \Omega$ with $\gamma(R) \in R$ for all $\emptyset \neq R \subseteq \Omega$. Now we define the value $\varphi$ as

$$
\varphi_{i}(v)=\sum_{i=\gamma(R)} \Delta_{v}(R)
$$

where $\Delta_{v}(R) \in \mathbb{R}$ is a Harsanyi dividend of the coalition $R \subseteq \Omega$ defined by $\Delta_{v}(R)=\sum_{T \subseteq R}(-1)^{|R|-|T|} v(T)$. It was shown in [6] that such values satisfy the axioms for quasi-values. 3 The cycle index of the trivial group is $Z\left(x_{1}\right)=x_{1}^{n}$ and substituting into (2) yields $\operatorname{dim} \mathcal{A}_{G_{1}}=n 2^{n-1}-2^{n}+1$. However, the number of selectors $\gamma: 2^{\Omega} \rightarrow \Omega$ is much larger, so many of the quasi-values defined by (5) are affine dependent 4

Example 2. ("Caste system") The set $\Omega$ is split into $k$ nonempty disjoint subsets ("castes") $\Omega_{1}, \ldots, \Omega_{k}$ and $G_{2}$ is chosen so that it guarantees equity within each $\Omega_{i}$. Formally, $G_{2}=\left\{\pi \in S_{\Omega} \mid \forall i \pi\left(\Omega_{i}\right)=\Omega_{i}\right\}$.

Some examples of $G_{2}$-symmetric quasivalues have been described in the literature. The Owen value, defined in [20, can be obtained as the expected value

\footnotetext{
${ }^{3}$ In the matrix representation, such values correspond to matrices $a_{i R}=\delta_{i \gamma(R)}$.

4 For $n \geq 4, \operatorname{dim} \mathcal{A}_{G_{1}}$ is strictly smaller than $n$ ! -1 which implies that the set of marginal operators (defined in Section 4.2) is also affine dependent.
} 
of marginal operators (see Section 4.2), if we first randomely choose an order of the castes and then the order of the players within each caste. Another related concept is the weighted Shapley value, studied by Kalai and Samet in [14. Here an order of the castes is given and within each caste, the profit is diveded among players proportional to their weights. In the case of equal weights of all players, the weighted Shapley value is symmetric with respect to all $G_{2}$-permutations.

The cycle index is $Z_{G_{2}}=\prod_{r=1}^{k} Z_{S_{\Omega_{r}}}$. We know from the proof of Theorem 2 that $|\chi / G|=\frac{1}{|G|} \sum_{g} 2^{\# c y c l e s(g)}$ for each set $\chi$ with a $G$-action. In particular, for $G=S_{n},\left|2^{\Omega} / G\right|=n+1$, because $S_{n}$-orbits of $2^{\Omega}$ are $O_{s}=\{R \subseteq \Omega|| R \mid=s\}$ for $s=0,1, \ldots, n$. This enables as to calculate

$$
\left.Z_{S_{n}}\right|_{(2, \ldots, 2)}=\frac{1}{n !} \sum_{\pi \in S_{n}} 2^{j_{1}(\pi)+\ldots+j_{n}(\pi)}=\frac{1}{n !} \sum_{\pi \in S_{n}} 2^{\# \operatorname{cycles}(\pi)}=\left|2^{\Omega} / S_{n}\right|=n+1 .
$$

If $G=S_{n}$, then the Shapley value is the only game value, so it follows from Theorem 2 that $\left.\left(\frac{d Z_{S_{n}}}{d x_{1}}-Z_{S_{n}}\right)\right|_{(2, \ldots, 2)}+1=0$ and $\left.\frac{d Z_{S_{n}}}{d x_{1}}\right|_{(2, \ldots, 2)}=n$. So, for $G_{2}=\prod_{r=1}^{k} S_{\Omega_{r}}$

$$
\left.\frac{d Z_{G_{2}}}{d x_{1}}\right|_{(2,2 \ldots 2)}=\left.\left(\sum_{r=1}^{k} \frac{d Z_{S_{\Omega_{r}}}}{d x_{1}} \prod_{s \neq r} Z_{S_{\Omega_{s}}}\right)\right|_{(2,2 \ldots 2)}=\sum_{r=1}^{k}\left|\Omega_{r}\right| \prod_{s \neq r}\left(1+\left|\Omega_{s}\right|\right)
$$

and

$$
\operatorname{dim} \mathcal{A}_{G_{2}}=\left(\sum_{r=1}^{k} \frac{\left|\Omega_{r}\right|}{1+\left|\Omega_{r}\right|}-1\right) \prod_{r=1}^{k}\left(1+\left|\Omega_{r}\right|\right)+1 .
$$

For the case of two castes $k=2$ this simplifies to $\left|\Omega_{1}\right| \times\left|\Omega_{2}\right|$.

Example 3. (Cyclic group) This example illustrates that transitive group action does not imply a unique $G$-symmetric quasi-value. If $G_{3}$ is the cyclic group $C_{n} \subseteq S_{n}$, the cycle index is $Z_{C_{n}}=\frac{1}{n} \sum_{f \mid n} \phi(f) x_{f}^{n / f}$, where $\phi(f)$ is the Euler totient function $\phi(f)=p_{1}^{k_{1}-1}\left(p_{1}-1\right) \ldots p_{r}^{k_{r}-1}\left(p_{r}-1\right)$, where $f=p_{1}^{k_{1}} \ldots p_{r}^{k_{r}}$ is the prime number decomposition. [8, p. 86]. Substituting into the formula in Theorem 2 gives

$$
\operatorname{dim} \mathcal{A}_{G_{3}}=2^{n-1}-\frac{1}{n} \sum_{f \mid n} \phi(f) 2^{n / f}+1 .
$$

In the case of $n=3$, the dimension turns out to be $2^{2}-\frac{1}{3}\left(2^{3}+2 \times 2\right)+1=1$, so there exists a one-dimensional space of quasi-values symmetric with respect to cyclic permutations of players.

\subsection{Shapley-value as an expected value of non-uniformly distributed marginal vectors}

Suppose that $\Omega=\{1,2, \ldots, n\}$, i.e. an order is given on the set of player. For a game $v \in \Gamma$ and a permutation $\pi \in S_{n}$, we may define a quasi-value $m_{\pi}$ by 


$$
\begin{aligned}
& \left(m_{\pi}\right)(v)_{\pi(1)}=v(\pi(1)) \text { and } \\
& \quad\left(m_{\pi}(v)\right)_{\pi(i)}=v(\{\pi(1), \pi(2), \ldots, \pi(i)\})-v(\{\pi(1), \pi(2), \ldots, \pi(i-1)\})
\end{aligned}
$$

for $i=2, \ldots, n$. We call $m_{\pi}$ the marginal operator and $m_{\pi}(v)$ the marginal vector [2, p. 19]. It corresponds to a situation where the players arrive in the order $\pi(1), \pi(2), \ldots, \pi(n)$ and each player is assigned the value of his or her contribution to the coalition of all players that have arrived before. The evaluation of $m_{\pi}$ on a game $u_{R}$ from the unanimity basis is $m_{\pi}\left(u_{R}\right)(\{\pi(i)\})=$ $u_{R}(\pi(1), \ldots, \pi(i))-u_{R}(\pi(1), \ldots, \pi(i-1))$ which is equal to 1 if and only if $\pi(i) \in R$ and $\pi(j) \notin R$ for $j>i$ and 0 otherwise. After the identification 1 we can represent $m_{\pi}$ is as a matrix

$$
\left(m_{\pi}\right)_{i R}=\left\{\begin{array}{l}
1 \text { iff } i \in R \text { and } \pi^{-1}(i)=\max \pi^{-1}(R) \\
0 \text { otherwise. }
\end{array}\right.
$$

A theorem of Weber [23] shows that if $\pi$ is a random permutation taken from a uniform distribution on $S_{n}$ then for any game $v$, the expected value of a marginal operator $m_{\pi}$ is the Shapley value. This can be generalized to the following statement.

Proposition 1. Let $G$ be a subgroup of $S_{n}$ and $A^{\pi}$ be a probability distributioin on $S_{n}$ constant on the right cosets $\{G \cdot \pi\}_{\pi}$, i.e. $A^{\pi}=A^{g \pi}$ for all $g \in G$ and $\pi \in S_{n}$. Then $\sum A^{\pi} m_{\pi}$ is a $G$-symmetric quasi-value.

Proof. We will show that the identity holds if evaluated on games from the unanimity basis of $\Gamma$. For the game $u_{R}$ (Definition 3), we start with the following equation:

$$
\left(g \cdot m_{\pi}\right)\left(u_{R}\right)=m_{g \pi}\left(u_{g R}\right)
$$

To prove this, we evaluate both sides on $\{i\}$ and rewrite the left-hand side to the equivalent equation

$$
\left(m_{\pi}\left(u_{R}\right)\right)\left(\left\{g^{-1}(i)\right\}\right)=\left(m_{g \pi}\left(u_{g R}\right)\right)(\{i\}) .
$$

Both sides are equal to 1 if and only if $\pi^{-1}\left(g^{-1}(i)\right)=\max \pi^{-1}(R)$ and 0 otherwise, which proves (6) for all $R \subseteq \Omega, i \in \Omega$ and $g \in G$. The $G$-symmetry of $\sum_{\pi \in S_{n}} A^{\pi} m_{\pi}$ follows from

$$
\begin{aligned}
& \left(g \cdot \sum_{\pi \in S_{n}} A^{\pi} m_{\pi}\right)\left(u_{R}\right)=\sum_{\pi \in S_{n}} A^{\pi}\left(g \cdot m_{\pi}\right)\left(u_{R}\right)=\sum_{\pi \in S_{n}} A^{\pi} m_{g \pi}\left(u_{g R}\right)= \\
& =\sum_{\pi \in S_{n}} A^{g \pi} m_{g \pi}\left(u_{g R}\right)=\sum_{g \pi=\pi^{\prime} \in S_{n}} A^{\pi^{\prime}} m_{\pi^{\prime}}\left(g \cdot u_{R}\right)=\left(\left(\sum_{\pi^{\prime} \in S_{n}} A^{\pi^{\prime}} m_{\pi^{\prime}}\right) \cdot g\right)\left(u_{R}\right)
\end{aligned}
$$

where we used (6) in the second and $A^{\pi}=A^{g \pi}$ in the third equality.

An immediate consequence of the classification Theorem 2 is that for $|\Omega|>3$ any quasi-value symmetric with respect to the alternating group $A_{n}$ is already 
the Shapley value. It follows from the last proposition that $\sum_{\pi} A^{\pi} m_{\pi}$ is the Shapley value not only for $A^{\pi}=\frac{1}{n !}$ but also for $A^{\pi}=\frac{s}{n !}$ for $\pi$ even and $A^{\pi}=\frac{2-s}{n !}$ for $\pi$ odd, $s \in[0,2]$. In fact, there are many other possibilities how to express the Shapley value as a convex combination of marginal operators. The space of all quasi-values on $\Omega$ is $\left(n 2^{n-1}-2^{n}+1\right)$-dimensional and the set of all probability distributions on $S_{n}$ is a $(n !-1)$-dimensional convex region in $\mathbb{R}^{n !}$, so there are at least $n !-n 2^{n}+2^{n-1}-2$ degrees of freedom for the choice of a distribution $A^{\pi}$ such that $\sum_{\pi} A^{\pi} m_{\pi}=$ Shapley.

Exponentially many (with respect to $n$ ) of these probability distributions $A^{\pi}$ can be constructed as follows. Choose $\Omega_{0} \subseteq \Omega,\left|\Omega_{0}\right|>3$ and define $S_{0}$ to be a group of all permutations $\pi$ acting identically on $\Omega \backslash \Omega_{0}$. Choose $\alpha \in(0,2)$ and define a probability distribution on $S_{n}$ by

$$
A^{\pi}\left(\Omega_{0}\right)=\left\{\begin{array}{l}
\frac{1}{n !} \text { if } \pi \notin S_{0} \\
\frac{\alpha}{n !} \text { if } \pi \in S_{0} \text { and } \pi \text { is even } \\
\frac{2-\alpha}{n !} \text { if } \pi \in S_{0} \text { and } \pi \text { is odd }
\end{array}\right.
$$

One can verify that the corresponding expected value of marginal operators $m_{\pi}$ is the Shapley value. For a set $\left\{\Omega_{1}, \Omega_{2}, \ldots, \Omega_{k}\right\}$ s.t. $\Omega_{i} \nsubseteq \Omega_{j}$ for all $i$ and $j$, the vectors $\left(A^{\pi}\left(\Omega_{i}\right)-\frac{1}{n !}\right)_{i} \in \mathbb{R}^{n !}$ are linearly independent and the distributions $\left(A^{\pi}\left(\Omega_{i}\right)\right)_{i}$ are affine independent.

\section{$5 \quad$ Appendix}

Here we finish the proof of Theorem 2 by the classification of supertransitive groups. Our proof is based on a classification of set-transitive permutation groups given by Beamont and Peterson in 1955 [1]. Another proof of the supertransitive groups classification was given by Michal Jordan on mathoverflow [13].

Theorem 3. $G$ is a supertransitive subgroup of $S_{n}$ if and only if one of the following conditions holds:

- $G$ is the full symmetric group $S_{n}$ for some $n$,

- $G$ is the alternating group $A_{n}$ for $n>3$,

- $G$ is conjugate to the image of an exotic embedding of $S_{5}$ to $S_{6}$.

Proof. Let $G \subseteq S_{n}$ be a group of permutations acting supertransitively on $\{1, \ldots, n\}$. This means that the stabilizer of each $A \subseteq\{1, \ldots, n\}$ acts transitively on $A$. Let $B \subseteq\{1, \ldots, n\}$ and $i, j \notin B$. Then $G$ acts transitively on $B \cup\{i, j\}$ and there exists a permutation $\pi \in G$ taking $B \cup\{i\}$ to $B \cup\{j\}$ such that $\pi(i)=j$. This implies that for each $A$ and $B$ s.t. $|A|=|B|>1$, there exists a permutation $\pi \in G$ s.t. $\pi(A)=B$. If $|A|=|B|=1$, the same is true because supertransitivity implies transitivity. We have shown that if the action of $G$ is supertransitive, it is also set-transitive.

If $G$ has a supertransitive action on $\{1, \ldots, n\}$, then its order has to be divisible by each $k \leq n$, because each $k$-element set $A$ is isomorphic to $G / G_{A}$, 
hence $|G|=|A| \times\left|G_{A}\right|$. So, $G$ has to be divisible by the least common multiple of $\{1, \ldots, n\}$.

Beamont and Petrson classified all set-transitive permutation groups in [1. It follows that such subgroups of $S_{n}$ are exactly the full symmetric group $S_{n}$ for any $n$, the alternating group $A_{n}$ for $n>2$ and 5 exceptions. The first and second exceptions are subgroups of $S_{5}$ of order 10, resp. 20. These groups cannot have a supertransitive action on $\{1, \ldots, 5\}$, because the lowest common multiple of $\{1, \ldots, 5\}$ is 60 . Two other exceptions in Beamont's classification are subgroups of $S_{9}$ of orders 504 and 1512 . These numbers are not divisible by the lowest common multiple of $\{1, \ldots, 9\}$ so we can exclude them as well. The last exception is a subgroup of $S_{6}$ of order 120 . This groups is equivalent to the exotic embedding of $S_{5}$ to $S_{6}$ and we will show that it acts supertransitively on $S_{6}$.

In [12], the authors realize this group action on $\{1, \ldots, 6\}$ as the conjugate action of $S_{5}$ on its six Sylow 5 -subgroups. Using this realisation, we may show that this action is supertransitive by direct calculation. Let as denote the Sylow 5 -subgroups by $I=\langle(12345)\rangle, I I=\langle(12354)\rangle, I I I=\langle(12435)\rangle, I V=\langle(12453)\rangle$, $V=\langle(12534))\rangle$ and $V I=\langle(12543)\rangle$. An elementary calculation shows that the image of a transposition in $S_{5}$ is the product of three disjoint transpositions in $S_{6}$, e.g. $(1,2) \in S_{5} \mapsto(I, V I)(I I, I V)(I I I, V)$ in the above realisation. Together with the set-transitivity of this $S_{5}$-action, this implies 2-supertransitivity. The image of a 3-cycle in $S_{5}$ is a product of two disjoint 3-cycles in $S_{6}$, which implies 3 -supertransitivity. Similarly, the image of a 4-, resp. 5-cycle in $S_{5}$ is a 4-, resp. 5 -cycle in $S_{6}$, which implies 4 - and 5-supertransitivity.

It remains to prove that $A_{n}$ is supertransitive if and only if $n>3$. First note that $A_{2}=\{i d\}$, reps. $A_{3}=\langle(123)\rangle$ are not supertransitive, because no element of these groups takes 1 to 2 and preserves $\{1,2\}$. Let $n>3$ and $A \subseteq\{1, \ldots, n\}$ be a $k$-set. If $k<n-1$, then any permutation of $A$ can be extended to an even permutation of $\{1, \ldots, n\}$. If $k=n-1>2$, then for each $i, j \in A$, there exists an even permutation of $A$ taking $i$ to $j$. This can be extended to an even permutation of $\{1, \ldots, n\}$, acting identically on the complement of $A$.

\section{Acknowledgements}

We would like to thank to Michal Jordan for his mathematical remarks and discussion on mathoverflow. This work was supported by MŠMT project number OC10048 and by the institutional research plan AV0Z100300504 and by

the Excelence project P402/12/G097 DYME Dynamic Models in Economics of GAČR.

\section{References}

1. R. Beaumont and R. Peterson. Set-transitive permutation groups. Canadian Journal of Mathematics, 7(1):35-42, 1955. 
2. R. Brânzei, D. Dimitrov, and S. Tijs. Models in Cooperative Game Theory: Crisp, Fuzzy, And Multi-Choice Games. Lecture notes in economics and mathematical systems. Springer Verlag, 2005.

3. R. Brink. An axiomatization of the shapley value using a fairness property. International Journal of Game Theory, 30:309-319, 2002.

4. S. Carnahan. Small finite sets, 2007.

5. S. David. The nucleolus of a characteristic function game. Siam journal on applied mathematics, 17(6):1163-1166, 1969.

6. J. Derks, H. Haller, and H. Peters. The selectope for cooperative games. Open access publications from maastricht university, Maastricht University, 2000.

7. J. Dixon and B. Mortimer. Permutation groups. Springer, 1996.

8. P. Flajolet and R. Sedgewick. Analytic combinatorics. Cambridge University Press, 2009.

9. J. Fulman. Cycle indices for the finite classical groups, 1997.

10. I. Gilboa and D. Monderer. Quasi-value on subspaces. International Journal of Game Theory, 19(4):353-363, 1991.

11. R. Gilles. The Cooperative Game Theory of Networks and Hierarchies. Theory and decision library: Game theory, mathematical programming, and operations research. Springer, 2010.

12. G. Janusz and J. Rotman. Outer automorphisms of $S_{6}$. The American Mathematical Monthly, 89(6):407410, 1982.

13. M. Jordan. Super-transitive group action (mathoverflow contribution). http://mathoverflow.net/questions/71917.

14. E. Kalai and D. Samet. On Weighted Shapley Values. International Journal of Game Theory, 16(3):205-222, 1987.

15. A. Kar. Axiomatization of the shapley value on minimum cost spanning tree games. Games and Economic Behavior, 38(2):265 - 277, 2002.

16. D. Monderer and W. H. Ruckle. On the Symmetry Axiom for Values of Nonatomic Games. Int. Journal of Math. And Math. Sci, 13(1):165-170, 1990.

17. J. Neumann, O. Morgenstern, A. Rubinstein, and H. Kuhn. Theory of Games and Economic Behavior. Princeton Classic Editions. Princeton University Press, 2007.

18. A. Neyman. Uniqueness of the shapley value. Games and Economic Behavior, 1(1):116 - 118, 1989.

19. A. Neyman. Values of Games with Infinitely Many Players. volume 3 of Handbook of Game Theory with Economic Applications (Chapt. 56), pages $2121-2167$. Elsevier, 2002.

20. G. Owen. Values of Games with A Priori Unions. In R. Henn and O. Moeschlin, editors, Mathematical Economics and Game Theory, volume 141 of Lecture Notes in Economics and Mathematical Systems, pages 76-88. Springer Berlin Heidelberg, 1977.

21. J. Rotman. An introduction to the theory of groups. Springer, 1995.

22. L. S. Shapley. A value for n-person games. Annals of Mathematics Studies, 2(28):307-317, 1953.

23. R. Weber. Probabilistic Values of Games. In A. Roth, editor, The Shapley value: essays in honor of Lloyd S. Shapley, pages 101-120. Cambridge Univ. Press, 1988.

24. H. P. Young. Monotonic solutions of cooperative games. International Journal of Game Theory, 14:65-72, 1985. 